\title{
DIAMETERS OF CONVEX BODIES
}

\author{
P. C. HAMMER
}

In [2], we have shown that the extended diameter family of a convex body $C$ in $n$-space has the property of outward simplicity if and only if there is no pair of parallel line segments in the boundary of $C$ respectively in an opposite pair of parallel contact planes of $C$. In [3] Professor Sobczyk and I show that every outwardly simple family of lines in the plane is the extended diameter family of a constant breadth convex body and incidentally give a simple construction for all constant breadth planar convex bodies. The purpose of this note is to show that not every continuous outwardly simple line family in Euclidean 3-space is the extended diameter family of a convex body.

A diameter of a convex body in $E_{n}(n \geqq 2)$ is a longest chord in its direction. In [2] it is proved that the diameters of a convex body cover the body.

Theorem 1. If $x$ is an interior point of a convex body $C$ such that every chord through $x$ is a diameter of $C$, then $C$ has $x$ as a center of symmetry.

Proof. Let $B$ be the intersection of any 2-flat containing $x$ with $C$. Then $B$ is a planar convex body and every chord of $B$ through $x$ is a diameter of $B$. We claim that $x$ bisects every diameter of $B$ through it. For let $B^{\prime}$ be the reflection of $B$ through $x$. Let $\beta^{\prime}$ and $\beta$ be the respective boundaries of $B^{\prime}$ and $B$. Then, since $x$ is interior to $B, \beta^{\prime}$ and $\beta$ intersect in at least two points. Now let $\rho_{1}=\rho(\theta)$ represent $\beta$ in a polar coordinate system with pole at $x$. Then $\rho_{2}(\theta)=\rho(\theta+\pi)$ represents $\beta^{\prime}$. Moreover, since the lines through $x$ contain diameters of $B$, there is a unique tangent line at all except possibly a denumerable number of points of $\beta$ and such a line is parallel to the opposite tangent line when it is unique. Hence

$$
\frac{\rho_{2}^{\prime}}{\rho_{2}}=\frac{\rho_{1}^{\prime}}{\rho_{1}}
$$

at all except possibly a denumerable number of points at which the difference quotients of $\rho_{1}$ and $\rho_{2}$ are bounded. Hence $\rho_{1}=c \rho_{2}$ since $\rho_{1}$ and $\rho_{2}$ are continuous and $c=1$ since $\beta^{\prime}$ and $\beta$ intersect. Hence $B^{\prime}=B$ and $x$ is a center point of $B$. Hence $x$ is a center point of $C$. This

\footnotetext{
Presented to the Society, June 21, 1952; received by the editors August 1, 1953.
} 
proves the theorem. Note that the requirement that $x$ be an interior point is needed since every proper chord of a convex cone through its vertex is a diameter of the cone.

A family $F$ of lines in $E_{n}$ is outwardly simple if and only if there exists an $(n-1)$-sphere $S$ such that $F$ covers $S$ and its exterior including infinite points simply and each line of $F$ pierces $S$ in two distinct points such that the interchange of these points results in a continuous involutory transformation of $S$ onto itself (without fixed point). See [1] for an associated result.

In $E_{2}$ it has been shown [3] that there is a constant breadth convex body with diameters extending to give any specified outwardly simple line family. We now describe an outwardly simple family in $E_{3}$ which is the extended diameter family of no convex body. Using, now, the customary coordinate system, let the line family $F$ contain a planar pencil of lines in each plane in a co-axial sheaf with the $x$-axis as axis. Let the dihedral angle made by one of these planes with the $x y$-plane be $\theta$. Let the focus of the planar pencil in that plane be $(\sin \theta, 0,0), 0 \leqq \theta<\pi$. Then, this family, $F$, of lines is clearly outwardly simple in $E_{3}$. However, suppose there were a convex body $C$ with a diameter along each line of $F$. From [2] an extension of these diameters by any ratio $r>1$ about the midpoint of each yields diameters of a larger convex body. Hence we may suppose that $C$ contains the closed line segment from $(0,0,0)$ to $(1,0,0)$ in its interior. Let $(a, 0,0)$ be a point on the boundary of $C$. We may take $a \geqq 2$. Then the sections of $C$ by the planes in the coaxial sheaf give planar convex bodies each of which has a pencil of diameters and hence a center point by Theorem 1 . But then, since these centers move on the $x$-axis the boundaries of the convex planar sections of $C$ cannot intersect on the negative axis. Hence, it is impossible to have a closed convex surface bounding a convex body with diameters extending to $F$.

This example leads to the following result.

THEOREM 2. If an outwardly simple line family $F$ in $E_{3}$ is a subfamily of the extended diameter family of a convex body, it cannot contain two distinct planar pencils of lines which are not confocal.

Proof. Suppose the theorem to be false; then there are two distinct planar pencils in $F$ not confocal and $F$ is the extended diameter family (or subfamily) of a convex body $C$. Then the planes of the two planar pencils cannot be parallel or points at infinity would be doubly covered by $F$. Hence the planes intersect in a line $m$ which must be in each pencil since the direction of $m$ can be "covered" but 
once. Hence we now have two distinct points on $m$ as foci of two pencils and the same argument as given above shows that no closed convex surface can have diameters extending along two such pencils.

The proof of Theorem 3 is similar.

THEOREM 3. An outwardly simple family $F$ of lines containing two distinct nonconfocal pencils each respectively in and covering an $(n-1)$ flat is not a subfamily of the extended diameter family of any convex body in $E_{n}$.

In [4] we give a characterization of all outwardly simple line families in $E_{n}, n>2$, which are the extended diameter families of constant breadth convex bodies. The characterization of line families which are the extended diameter families of any convex body we have not accomplished for $n>2$.

\section{REFERENCES}

1. Amasa Forrester, $A$ theorem on involutory transformations without fixed points, Proc. Amer. Math. Soc. vol. 3 (1952) pp. 333-334.

2. P. C. Hammer, Associated convex bodies, unpublished manuscript.

3. P. C. Hammer and Andrew Sobczyk, Planar line families. I, Proc. Amer. Math. Soc. vol. 4 (1953) pp. 226-233.

4. P. C. Hammer, Constant breadth convex surfaces, unpublished manuscript.

Los Alamos Scientific Laboratory 